\title{
Association of bone morphogenetic protein-2 gene polymorphisms with susceptibility to ossification of the posterior longitudinal ligament of the spine and its severity in Chinese patients
}

\author{
Hao Wang $\cdot$ Dongmei Liu $\cdot$ Zhaohui Yang · \\ Baopeng Tian · Jie Li $\cdot$ Xianglong Meng • \\ Zhentian Wang $\cdot$ Hui Yang $\cdot$ Xin Lin
}

Received: 23 August 2007/Revised: 31 January 2008/Accepted: 4 March 2008/Published online: 4 April 2008

(C) The Author(s) 2008

\begin{abstract}
A case-control study was conducted to examine the association between two single nucleotide polymorphisms (SNPs) in exon 2 of the bone morphogenetic protein-2 gene (BMP-2) and ossification of the posterior longitudinal ligament (OPLL), and to investigate whether SNPs of the Ser37Ala (T/G) and the Ser87Ser $(\mathrm{A} / \mathrm{G})$ in the BMP-2 gene are associated with genetic susceptibility to OPLL and its severity in Chinese subjects. The Ser87Ser (A/G) SNP has been implicated
\end{abstract}

H. Wang $\cdot$ B. Tian $\cdot$ X. Lin $(\varangle)$

Beijing Tiantan Hospital, Capital Medical University (CMU),

Beijing 100050, China

e-mail: linxin@bjtth.com

H. Wang

e-mail: haowang80@163.com

D. Liu $\cdot$ H. Yang $(\bowtie)$

Beijing Institute for Neuroscience,

Beijing Center of Neural Regeneration and Repair,

Key Laboratory for Neurodegenerative Disease of the Ministry

of Education, Capital Medical University (CMU),

Beijing 100069, China

e-mail: huiyang@ccmu.edu.cn

Z. Yang

Second Hospital, Shanxi Medical University,

Taiyuan 030001, China

J. Li

Dianli Hospital, Beijing 100073, China

X. Meng

Chaoyang Hospital, Capital Medical University (CMU),

Beijing 100020, China

Z. Wang

Center Hospital, Chaoyang 100021, China in bone mineral density (BMD) and increases the risk of $\mathrm{OA}$ in women. The Ser37Ala (T/G) SNP is associated with BMD and the rate of bone loss in osteoporosis and osteoporosis fractures. A total of 57 OPLL patients and 135 non-OPLL controls were studied. Radiographs of the cervical spine were analyzed to determine the presence and the severity of OPLL. The association of two SNPs with the occurrence and the extent of OPLL were statistically evaluated. There was a significant association between the Ser37Ala (T/G) polymorphism and the occurrence of OPLL in the cervical spine. However, no significant association was found between the Ser37Ala (T/G) polymorphism and the more number of ossified cervical vertebrae in OPLL patients. There was a significant association between the Ser87Ser (A/G) polymorphism and the more number of ossified cervical vertebrae in OPLL patients. However, there was no statistical difference between the Ser87Ser (A/G) SNP and the occurrence of OPLL in the cervical spine. In addition, the Ser87Ser $(\mathrm{A} / \mathrm{G})$ polymorphism in male patients and in female patients showed no statistical difference between cases and controls. The present results demonstrate that BMP-2 Gene is not only a factor associated with the occurrence of OPLL, but also a factor related to more extensive OPLL. The "G" allele in the Ser37Ala (T/G) polymorphism is associated with the occurrence of OPLL, but not more extensive OPLL in the cervical spine. The "G" allele in the Ser87Ser $(A / G)$ polymorphism promotes the extent of OPLL, whereas the "A" allele in the Ser87Ser (A/G) polymorphism restricts ectopic ossification in the cervical spine at least in Chinese subjects.

Keywords OPLL · BMP-2 gene · PCR · Polymorphism · Case-control study 


\section{Introduction}

Ossification of the posterior longitudinal ligament (OPLL) has a strong genetic component, based on nation-wide pedigree surveys [30], surveys of twins [26], and HLA haplotype analysis [15, 22]. Because genetic factors appear to have a crucial role in OPLL, the use of molecular genetic studies is important to the understanding of the molecular etiologies of OPLL and will lead to the development of new therapeutics. However, multiple genetic and environmental components must contribute to the development of OPLL. Therefore, it has been difficult to clarify all the susceptible genes for OPLL. OPLL of the spine was firstly reported in Japan and is a common disorder among Japanese and other Asian populations. The prevalence of OPLL in Japan is $1.9-4.3 \%$ of the general population $>30$ years old [14]. OPLL has a prevalence of $0.44-8.92 \%$ and mean prevalence of $3.08 \%$ among Chinese [8]. Heterotopic Ossification of the spinal ligament is the specific feature of OPLL that causes compression of the spinal cord and leads to various degrees of myelopathy.

The previous studies described linkage evidence in the HLA region of chromosome 6 in 91 affected sib pairs and identified the collagen 11A2 (COL11A2) gene as a possible candidate [7]. Gender-specific associations indicated that genetic factors involving COL11A2 play a specific role in the etiology of OPLL exclusively in males [11]. Allelic association studies between OPLL and molecular variants in COL11A2 demonstrated that a nucleotide substitution at intron 6(-4), a $\mathrm{T} \rightarrow \mathrm{A}$ substitution, is significantly associated with OPLL, and the functional variant results in altered splicing, which is protective in the pathogenesis of OPLL [10]. The extensive linkage disequilibrium and haplotype association studies have demonstrated that seven single nucleotide polymorphisms (SNPs) of COL6Al gene are associated with OPLL. Among them, the intron 32 (-29) SNP is most significantly associated with OPLL [28]. Nakamura et al reported that the nucleotide pyrophosphatase gene (NPPS), which has been identified as a cause in a mouse model of OPLL [18], is also associated with OPLL [16]. The results demonstrated that the IVS2011delT allele in the NPPS gene and the A861G polymorphism in the leptin receptor gene are associated with more extensive OPLL, but not with its occurrence rate [25].

Recently, many factors have been identified in bone matrix of OPLL, including transforming growth factor $\beta$ (TGF- $\beta$ ), Bone morphogenetic protein (BMP), fibroblast growth factor, and insulin-like growth factor $\alpha$. Among these factors, only BMP was shown to induce ectopic ossification when implanted subcutaneously [34]. Immunohistochemical studies using a monoclonal antibody against partially purified bovine BMP demonstrated that BMP was distributed in periosteal cells and mesenchymal cells of marrow stroma in normal human bone and chondrocytes and mesenchymal cells of the fracture site during repair. In addition, recombinant BMP-2 was reported to have a potent effect on stimulating differentiation of a mesenchymal cell line of osteoblast lineage to cells with osteoblastic phenotype as evidenced by the expression of osteocalcin mRNA [36]. From these results, it is likely that the primary effect of BMP is to initiate the ossification process by inducing differentiation mesenchymal progenitor cells to stimulate cartilage formation during the development of OPLL.

BMP-2 has been implicated as an important regulator of bone metabolism [5]. The immunohistochemical studies demonstrated that BMP- 2 and TGF- $\beta$ are present in ossifying matrix and chondrocytes are present adjacent to cartilaginous areas of OPLL tissues, and that BMP-2 is also localized in mesenchymal cells with fibroblastic features in the immediate vicinity of the cartilaginous areas, and that nonossified ligaments do not contain either of these growth factors. It is suggested that BMP-2 plays different roles in the different stages of development of ectopic ossification [5]. How the production of these factors is stimulated at the ossifying ligaments remains unclear. Further studies are needed to clarify the molecular mechanism of the development of ectopic ossification in OPLL.

Rather, several polymorphisms of the BMP-2 gene have been identified $[24,33]$. One of the polymorphisms is a $\mathrm{T} \rightarrow \mathrm{G}$ transition at nucleotide 116 in exon 2 of BMP-2, resulting in a Ser $\rightarrow$ Ala substitution at amino acid position 37. Another Ser87Ser $(A / G)$ polymorphism is samesense mutation. The Ser37Ala (T/G) and Ser87Ser (A/G) polymorphisms are, respectively, relative to Osteoporosis and Osteoarthritis disease [24, 33]. As a genetic disease associated with abnormal calcium phosphate metabolism [27], OPLL occurs with a greater frequency in genetically related metabolic diseases. The previous studies showed that the Ser87Ser (A/G) SNP has been implicated in bone mineral density (BMD) and increase the risk of $\mathrm{OA}$ in women [32, 33]. The Ser37Ala (T/G) SNP is associated with BMD and the rate of bone loss in osteoporosis and osteoporosis fractures [24].

The purpose of this study was to investigate association of two SNPs of BMP-2 gene with susceptibility to OPLL of the spine and its severity in Chinese patients. Association between two SNPs of BMP-2 gene and OPLL is firstly reported in the paper.

\section{Subjects and methods}

Disease criteria and subjects

The ethical committees of the Beijing Tiantan Hospital Capital Medical University approved the study. Informed 
consent was obtained from all the participants in this study. Records of 310 consecutive spine patients with spine diseases treated between May 2005 and January 2007 were reviewed for eligibility according to the inclusion criteria. Records of 57 patients met all criteria and formed the clinical subjects for this study. The participants in this study were 57 patients with OPLL and 135 control subjects without OPLL. All 192 participants are from the Beijing Tiantan Hospital Capital Medical University and live in Beijing region. The average age of the patients including 32 males and 25 females is 54.9 years. Controls were also collected on age-matched and sex-matched controls (74 males and 61 females). In addition, all 192 participants are from Han race of Chinese people in mainland, China. Therefore, the cases and controls are from the same study base and are genetically matched. Multiple authors including an orthopedic professor, an orthopedic surgery chief resident, and an orthopedic surgery junior resident performed the diagnosis of OPLL. The diagnosis of OPLL was based on radiological findings including radiographs, computed tomogram (CT), and magnetic resonance imaging (MRI) of the cervical spine according to the criteria reported by Tsuyama [31]. OPLL in the cervical spine was diagnosed in 57 participants: 21 with continuous type, 12 with mixed type, 19 with segmental type, and 5 with localized type. The severity of OPLL was determined by the number of ossified cervical vertebrae on these lateral radiograph films. Patients were also stratified according to the extent of ossification. The patients with ankylosing spondylitis and metabolic diseases associated with OPLL such as hypophosphate rickets/osteomalacia, diffuse idiopathic skeletal hyperostosis (DISH), and hyperparathyroidism were excluded according to radiographic and biochemical examinations. The patients with increased BMD and/or decreased BMD were also excluded. The most common reason for excluding a patient from the study was ankylosing spondylitis and metabolic diseases associated with OPLL. None of the control patients exhibited OPLL.

Genotyping and SNPs of the BMP-2 gene

Venous blood $(5 \mathrm{ml})$ was collected in tubes containing ethylenediaminetetraacetic acid (EDTA) (50 mmol/l of disodium salt), and genomic deoxyribonucleic acid (DNA) was isolated with Wizard Genomic DNA Purification Kit (produced by Promega Corpation of USA). For the BMP-2 gene, several SNPs were already reported $[24,33]$. We analyzed two of them (Ser37Ala T/G and Ser87Ser A/G in exon 2 of BMP-2 gene). Polymerase chain reaction (PCR) with two primers (a sense primer 5'-CTCACGTCGGT CCTGTCC- $3^{\prime}$ and an antisense primer $5^{\prime}$-CCCTG CTCCATGCCTCAC $-3^{\prime}$ ) was performed with a standard protocol. Reactions were performed in a total volume of $50 \mu \mathrm{l}$ containing $0.5 \mu \mathrm{g}$ of genomic DNA; $1 \mu \mathrm{l}$ of each primer $(20 \mu \mathrm{M}) ; 8 \mu \mathrm{l}$ dCTP, dTTP, dGTP, and dATP mixture (each $2.5 \mu \mathrm{M}$ ); $0.5 \mu \mathrm{l}$ TAKARA LA Taq DNA polymerase (produced by TakaRa Biotechnology dalian Co., Ltd); $5 \mu 1 \mathrm{MgCl}_{2}(25 \mathrm{mM}) ; 5 \mu \mathrm{l} 10 \times$ LA PCR Buffer II $\left(\mathrm{Mg}^{2+}\right.$ Free $)$; double distilled $\mathrm{H}_{2} \mathrm{O}\left(\mathrm{DDH}_{2} \mathrm{O}\right)$ up to $50 \mu \mathrm{l}$. The thermocycling procedure consisted of initial denaturation at $95^{\circ} \mathrm{C}$ for $5 \mathrm{~min}, 40$ cycles of denaturation at $95^{\circ} \mathrm{C}$ for $40 \mathrm{~s}$, annealing at $61^{\circ} \mathrm{C}$ for $40 \mathrm{~s}$, extension at $72^{\circ} \mathrm{C}$ for $40 \mathrm{~s}$, and a final extension at $72^{\circ} \mathrm{C}$ for $10 \mathrm{~min}$. The PCR products (including two SNPs in exon 2 of BMP-2 gene) were analyzed by direct sequencing using BigDye Terminator cycle sequencing on an ABI 3730XL POP7 DNA sequencing analysis 5.2 (Applied Biosystems). The expected size of the specific amplification product was 393 bp.

\section{Statistical analysis}

To assess the genotypic and allelic distribution, the Chisquare test (Fisher's exact test) was performed. To compare the number of ossified cervical vertebrae between two groups, the nonparametric test (Mann-Whitney exact test) was employed. A $P$ value less than 0.05 was considered statistically significant.

\section{Results}

The distribution of genotype and allele type of two SNPs within the BMP-2 gene in OPLL patients and controls is shown in Table 1. Two SNPs in exon 2 of the BMP-2 gene were accurately detected by direct sequencing using BigDye Terminator cycle sequencing on an ABI 3730XL POP7 DNA sequencing analysis 5.2 (Applied Biosystems, Figs. 1, 2).

Table 1 Genotypic and allelic distributions of two SNPs in BMP-2 gene between OPLL patients and non-OPLL Controls

\begin{tabular}{lllllll}
\hline SNPs & \multicolumn{3}{l}{ Ser37Ala $(\mathrm{T} / \mathrm{G})$} & \multicolumn{3}{l}{ Ser87Ser $(\mathrm{A} / \mathrm{G})$} \\
\hline Genotype & TT & TG & GG & AA & AG & GG \\
$\quad$ OPLL $(n=57)$ & 38 & 19 & 0 & 1 & 15 & 41 \\
$\quad$ Control $(n=135)$ & 126 & 9 & 0 & 0 & 23 & 112 \\
Fisher's exact test & $P<0.001$ & & $P=0.081$ & \\
Allele & T & G & A & G \\
$\quad$ OPLL & 95 & 19 & 17 & 97 \\
$\quad$ Control & 261 & 9 & 23 & 247 \\
Fisher's exact test & $P<0.001$ & & $P=0.069$ &
\end{tabular}

Note: Difference in genotypic and allelic distribution between subpopulation of OPLL patients and controls were analyzed using $\chi^{2}$-test 
Fig. 1 The plain radiographs and sequencing results of a 62-year-old man with OPLL. a The plain radiographs showed patient had ossification of the posterior longitudinal ligament in the cervical spine $(\mathrm{C} 2-\mathrm{C} 4$, C5-C6, C7 mixed type). b The PCR products showed the TG heterozygosis mutation of the Ser37Ala (T/G) polymorphism in exon 2 of the BMP-2 gene. A allele: green line; $\mathrm{T}$ allele: red line; $\mathrm{C}$ allele: blue line; $\mathrm{G}$ allele: black line direct sequencing result of the
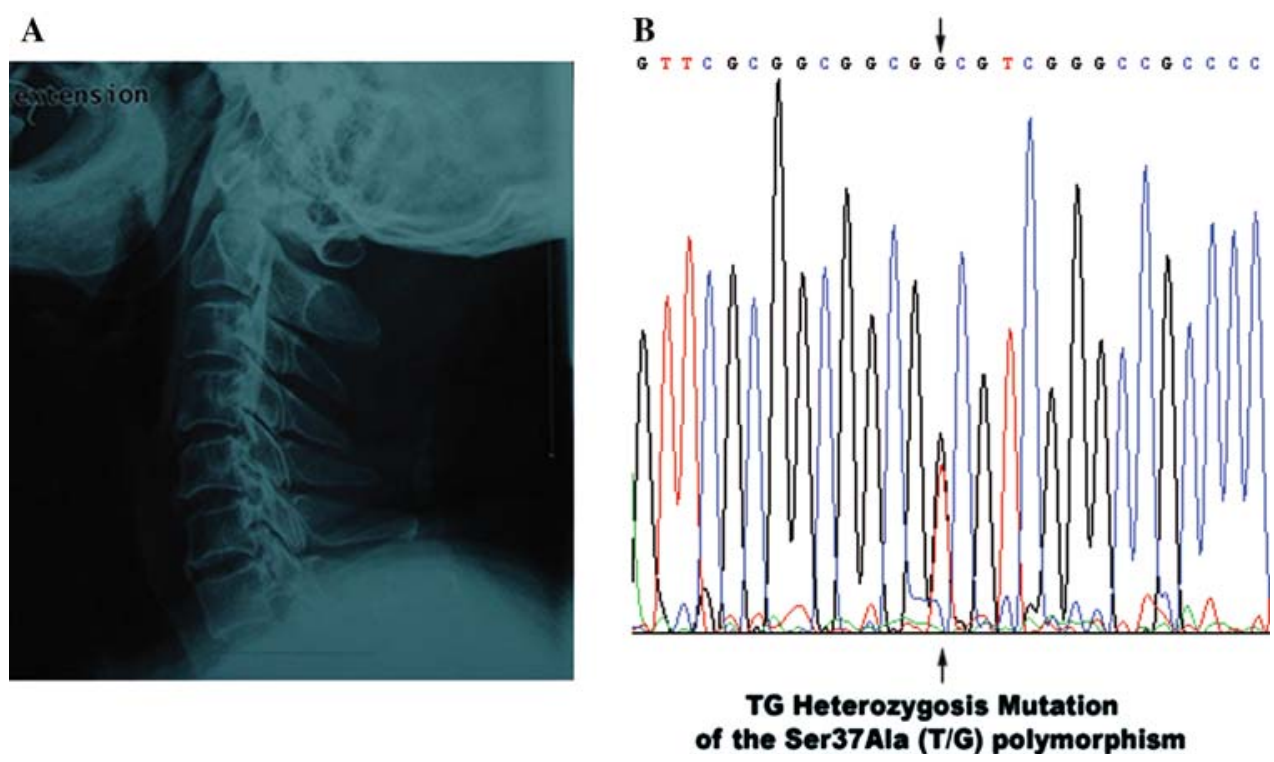

Fig. 2 The 2-dimensional (2D)-computerized tomography (CT) and sequencing results of a 50-year-old woman with OPLL. a The 2D-CT showed patient had ossification of the posterior longitudinal ligament in the cervical spine (C2-C3, C4, C5-C6 mixed type). b The direct sequencing result of the PCR products showed the AG heterozygosis mutation of the Ser87ser (A/G) polymorphism in exon 2 of the BMP-2 gene. $\mathrm{A}$ allele: green line; $\mathrm{T}$ allele: red line; $\mathrm{C}$ allele: blue line; $\mathrm{G}$ allele: black line

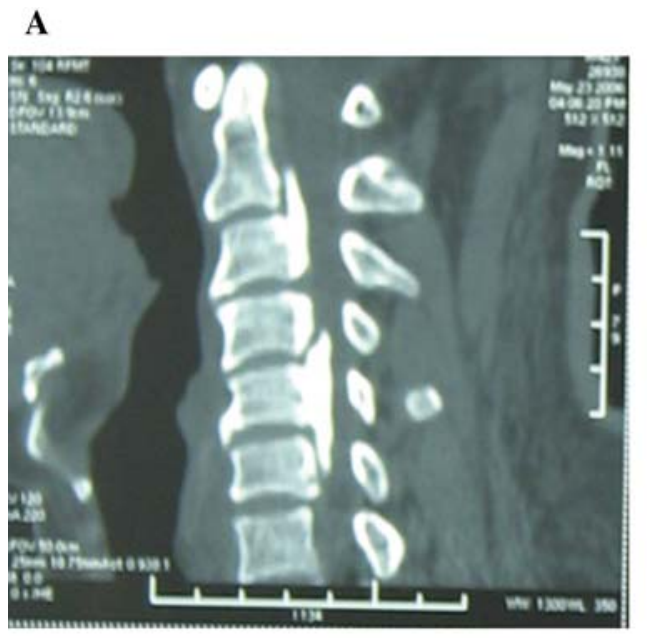

The numbers of the "TT," "TG," and "GG" genotypes of the Ser37Ala (T/G) in this study population were 164 , 28 , and 0 , and the frequencies were 85,15 , and $0 \%$, respectively. The numbers of the "AA," "AG," and "GG" genotypes of the Ser87Ser $(\mathrm{A} / \mathrm{G})$ in this study population were 1,38 , and 153 , and the frequencies were $0.005,19.79$, and 79.69 , respectively.

Comparing the distributions of genotype of two SNPs, there was statistical difference among the "TT," "TG," and "GG" in the Ser37Ala (T/G) polymorphism (Fisher's exact $P<0.001)$. The "TG" genotype is associated with the occurrence of OPLL. There was no statistical difference among the "TT," "TC," and "CC" genotypes in the Ser87Ser (A/G) polymorphism (Fisher's exact $P=0.081$ ). No association was found between male and female Ser87Ser (A/G) genotypes in cases (Fisher's exact $P=0.366)$.
Comparing the distributions of allele type of two SNPs, there was a significant association between the Ser37Ala (T/G) polymorphism and the occurrence of OPLL in the cervical spine. The studies show that the frequency of the "G" allele is significantly higher in subjects with OPLL than in control subjects (Fisher's exact $P<0.001$ ). However, there was no statistical difference between the Ser87Ser $(\mathrm{A} / \mathrm{G})$ polymorphism and the occurrence of OPLL in the cervical spine (Fisher's exact $P=0.069$ ). In addition, the Ser87Ser (A/G) SNP in male patients (Fisher's exact $P=0.102$ ) and female patients (Fisher's exact $P=0.117)$ of the cases was not significantly associated with controls. There were no allelic frequency differences between males and females in control groups (Fisher's exact $P=1.000)$. The Ser87Ser $(\mathrm{A} / \mathrm{G})$ polymorphism in male patients (Fisher's exact $P=0.155$ ) and in female patients (Fisher's exact $P=0.260$ ) also showed no 
statistical difference between cases and controls. Table 2 shows genotypic and allelic distributions of Ser87Ser $(\mathrm{A} / \mathrm{G})$ classified by gender in OPLL Patients and nonOPLL Controls.

The distribution of ossified cervical vertebrae of OPLL patients in each subgroup classified by genotype of two SNPs is shown in Tables 3 and 4. The authors further investigated the association of two SNPs with the number of ossified cervical vertebrae in OPLL patients (Figs. 3, 4). Regarding the Ser37Ala (T/G) polymorphism in the BMP-2 gene, Patients with the "G" allele (genotype, "TG" or "GG") in the polymorphism showed no significantly greater number of ossified cervical vertebrae than those without the "G" allele (genotype, TT; Mann-Whitney exact $P=0.113)$. The "G" allele in the Ser37Ala (T/G) polymorphism is not associated with the extent of heterotopic ossification in the cervical spine in OPLL patients. Regarding the Ser87Ser (A/G) polymorphism in the BMP2 gene, patients without the "A" allele (genotype, "GG") in the Ser87Ser $(A / G)$ polymorphism showed a significantly greater number of ossified cervical vertebrae than those with the "A" allele (genotype, "AG" or "AA"; Mann-Whitney exact $P<0.001)$. The present results indicate that the " $\mathrm{G}$ " allele promotes the extent of OPLL,

Table 2 Genotypic and allelic distributions of Ser87Ser (A/G) classified by gender in OPLL patients and non-OPLL Controls

\begin{tabular}{lcrr}
\hline SNPs & \multicolumn{2}{c}{ Ser87Ser $(\mathrm{A} / \mathrm{G})$} & \\
\hline Genotype & AA & AG & GG \\
OPLL $(n=57)$ & & & \\
Male $(n=32)$ & 0 & 10 & 22 \\
Female $(n=25)$ & 1 & 5 & 19 \\
Fisher's Exact Test & $P=0.366$ & & \\
Control $(n=135)$ & 0 & 23 & 112 \\
$\quad$ Male $(n=85)$ & 0 & 15 & 70 \\
$\quad$ Female $(n=50)$ & 0 & 8 & 42 \\
Allele & $\mathrm{G}$ & & $\mathrm{A}$ \\
OPLL & & & \\
$\quad$ Male & 54 & & 7 \\
$\quad$ Female & 43 & & 23 \\
Control & 247 & & 15 \\
$\quad$ Male & 155 & & 8 \\
Female & 92 & & \\
\hline
\end{tabular}

Note: Difference in genotypic and allelic distribution classified by gender between OPLL patients and controls were analyzed using $\chi^{2}$-test

Male of OPLL and control, Fisher's exact $P=0.102$

Male of OPLL and male of controls, Fisher's Exact $P=0.155$

Female of OPLL and control, Fisher's exact $P=0.117$

Female of OPLL and female of controls, Fisher's exact $P=0.260$

Male and female in controls, Fisher's exact test $=1.000$ whereas the "A" allele restricts ectopic ossification in the cervical spine in OPLL patients.

\section{Discussion}

OPLL of the spine is a subset of "bone-forming" diseases, characterized by ectopic ossification in the spine ligaments [19, 20, 31]. OPLL is a common disorder among elderly populations and is the leading cause of spinal myelopathy in Asian populations [14]. The pathogenesis of OPLL remains unknown. The etiologies of all complex diseases potentially involve gene-gene and gene-environment interactions. The OPLLs high concordance in twins [26]. and the association with particular human leukocyte antigen haplotypes $[15,22]$ suggested that genetic factors may be involved in its pathogenesis. The histopathologic features of OPLL are characterized by the abnormal expression of BMP-2, TGF- $\beta$, and Type I, II, and XI collagens (COL11) in the regional ligament [5, 37]. Therefore, multiple rather than single, dominant genes, including COL11, BMP-2, TGF- $\beta 1$, should be considered as candidate genes involved in the predisposition to OPLL. The previous studies showed that the restriction fragment length polymorphism (RFLP) of estrogen receptor (ER) gene, interleukin-1 (IL-1) gene, and the SNPs of TGF- $\beta$ gene, NPPS gene, Leptin Receptor gene, and COL11A2 gene are associated with the development of OPLL $[10,17$, 25].

BMP-2 is one of the members of TGF- $\beta$ superfamily and acts as a potent stimulator of bone formation in vivo. In addition, immunohistochemical studies demonstrated that BMP-2 and TGF- $\beta$ are present at ossifying matrix and chondrocytes of ossifying ligaments, and that only BMP-2 is localized at mesenchymal cells adjacent to these areas, and that nonossified ligament does not contain these growth factors. It is suggested that BMP-2 and TGF- $\beta$ might play an important role during the development of OPLL and that these factors play different roles at different stages of development of ectopic ossification [5].

BMP-2 gene, on chromosome 20p, contains three exons. Exon 1 comprises a $5^{\prime}$ untranslated region and exon 2 contains a hydrophobic leader sequence followed by 118 amino acids. Exon 3 encodes 283 amino acids, of which the terminal 114 amino acids constitute the mature protein [21, 35]. BMP-2 gene has been considered to be a likely candidate involved in the OPLL pathogenesis. However, a previous study reported no significant association between the RFLP of BMP-2 polymorphism and the prevalence of OPLL in the cervical spine. The authors concluded that two RFLPs of the BMP-2 allele were identified by digestion with MspI and TaqI, which revealed alleles of 3.0 and $0.6 \mathrm{~kb}$, and 2.5 and $2.1 \mathrm{~kb}$, respectively. The genotype 
Table 3 The distribution of ossified cervical vertebrae of OPLL patients in each subgroup classified by "G" allele of the Ser37Ala (T/G)

\begin{tabular}{|c|c|c|c|}
\hline \multicolumn{2}{|c|}{ Group $1=\mathrm{G}$ allele $(+)$} & \multicolumn{2}{|c|}{ Group $2=\mathrm{G}$ allele $(-)$} \\
\hline $\begin{array}{l}\text { Number of } \\
\text { OPLL } \\
\text { patients }\end{array}$ & $\begin{array}{l}\text { Number of } \\
\text { ossified } \\
\text { cervical } \\
\text { vertebrae }\end{array}$ & $\begin{array}{l}\text { Number of } \\
\text { OPLL patients }\end{array}$ & $\begin{array}{l}\text { Number of } \\
\text { ossified } \\
\text { cervical } \\
\text { vertebrae }\end{array}$ \\
\hline 1 & 2 & 1 & 1 \\
\hline 2 & 6 & 2 & 2 \\
\hline 3 & 3 & 3 & 3 \\
\hline 4 & 2 & 4 & 2 \\
\hline 5 & 2 & 5 & 1 \\
\hline 6 & 1 & 6 & 3 \\
\hline 7 & 3 & 7 & 2 \\
\hline 8 & 1 & 8 & 2 \\
\hline 9 & 2 & 9 & 5 \\
\hline 10 & 1 & 10 & 3 \\
\hline 11 & 1 & 11 & 4 \\
\hline 12 & 2 & 12 & 2 \\
\hline 13 & 2 & 13 & 3 \\
\hline 14 & 6 & 14 & 3 \\
\hline 15 & 1 & 15 & 2 \\
\hline \multirow[t]{22}{*}{16} & 3 & 16 & 3 \\
\hline & & 17 & 2 \\
\hline & & 18 & 1 \\
\hline & & 19 & 7 \\
\hline & & 20 & 7 \\
\hline & & 21 & 3 \\
\hline & & 22 & 4 \\
\hline & & 23 & 3 \\
\hline & & 24 & 1 \\
\hline & & 25 & 3 \\
\hline & & 26 & 2 \\
\hline & & 27 & 3 \\
\hline & & 28 & 2 \\
\hline & & 29 & 2 \\
\hline & & 30 & 5 \\
\hline & & 31 & 3 \\
\hline & & 32 & 3 \\
\hline & & 33 & 3 \\
\hline & & 34 & 2 \\
\hline & & 35 & 1 \\
\hline & & 36 & 2 \\
\hline & & 37 & 7 \\
\hline
\end{tabular}

frequency of MspI and TaqI alleles was not significantly different between the patients with OPLL and the control subjects [6]. However, their study included only 18 patients with OPLL and did not further research the single nucleotide polymorphisms of BMP-2 gene. In this study, oligonucleotide primers, the forward primer starting at nucleotide 122109 of the BMP-2 gene (Genbank accession number AL035668) and the reverse primer starting at nucleotide 122502 of the BMP-2 gene, amplify the BMP-2 gene mRNA sequence in exon 2 .

The current studies show that the Ser37Ala (T/G) polymorphism in exon 2 of BMP-2 gene is associated with the occurrence of OPLL, but not with more extensive OPLL in the cervical spine. The "TG" genotype is associated with the occurrence of OPLL. The results indicate that the "G" allele is a risk factor associated with the genetic susceptibility to OPLL, but not a factor related to the extent of heterotopic ossification in the cervical spine. Previously, the studies described that the Ser37Ala (T/G) polymorphism shows significant association with osteoporosis [24]. The present results demonstrate that the Ser37Ala (T/G) polymorphism in the BMP-2 gene initiates the ectopic ossification during the development of OPLL.

The present studies show that the Ser87Ser (A/G) polymorphism in the BMP-2 gene is associated with more extensive OPLL, but not with its occurrence rate in the cervical spine. The "G" allele in the Ser87Ser (A/G) polymorphism promotes the extent of OPLL, but does not promote an increased susceptibility to OPLL. Patients not carrying the "A" allele in the Ser87Ser (A/G) polymorphism showed a greater number of ossified vertebrae than the "A" allele-carrier patients. These results indicate that the " $G$ " allele promotes the extent of OPLL, whereas the "A" allele restricts ossification. Furthermore, the analysis adjusted for gender showed that the Ser87Ser (A/G) polymorphism in male patients and in female patients showed no statistical difference between the OPLL groups and the control groups. The present results demonstrate the Ser87Ser $(\mathrm{A} / \mathrm{G})$ polymorphism promotes the extent of ectopic ossification during the development of OPLL. The Ser87Ser (A/G) SNP is samesense mutation. The authors found that Chinese do not carry commonly the "AA" genotype but the "GG" or "AG" genotype in the Ser87Ser $(\mathrm{A} / \mathrm{G})$ polymorphism. Only one "AA" genotype was found in our samples. The patient with "AA" genotype showed ossification of the posterior longitudinal ligament in the cervical spine (C4, C5 segmental type). However, the patient did not have any clinical symptom. On the other hand, the "G" allele in the Ser87Ser (A/G) polymorphism is a risk genetic factor for the extent of OPLL in this study. Therefore, the results may be benefit to clarify that OPLL is a common disorder among Asian populations. In addition, the Gln89Glu $(\mathrm{C} / \mathrm{G})$ polymorphism was found by chance in this study. However, the present studies showed only one patient had the Gln89Glu (C/G) polymorphism in all samples. The patient with "CG" genotype heterozygosis mutation in the Gln89Glu (C/G) polymorphism showed the OPLL in the cervical spine (C2-C3, C4 mixed 
Table 4 The distribution of ossified cervical vertebrae of OPLL patients in each subgroup classified by "A" allele of the Ser87Ser (A/G)

\begin{tabular}{|c|c|c|c|}
\hline \multicolumn{2}{|c|}{ Group $1=$ A allele $(+)$} & \multicolumn{2}{|c|}{ Group $2=$ A allele $(-)$} \\
\hline $\begin{array}{l}\text { Number of } \\
\text { OPLL } \\
\text { patients }\end{array}$ & $\begin{array}{l}\text { Number of } \\
\text { ossified } \\
\text { cervical } \\
\text { vertebrae }\end{array}$ & $\begin{array}{l}\text { Number of } \\
\text { OPLL } \\
\text { patients }\end{array}$ & $\begin{array}{l}\text { Number of } \\
\text { ossified } \\
\text { cervical } \\
\text { vertebrae }\end{array}$ \\
\hline 1 & 2 & 1 & 1 \\
\hline 2 & 2 & 2 & 2 \\
\hline 3 & 2 & 3 & 3 \\
\hline 4 & 1 & 4 & 1 \\
\hline 5 & 2 & 5 & 3 \\
\hline 6 & 1 & 6 & 6 \\
\hline 7 & 2 & 7 & 2 \\
\hline 8 & 1 & 8 & 5 \\
\hline 9 & 2 & 9 & 3 \\
\hline 10 & 2 & 10 & 6 \\
\hline 11 & 2 & 11 & 2 \\
\hline 12 & 2 & 12 & 3 \\
\hline 13 & 2 & 13 & 3 \\
\hline 14 & 1 & 14 & 3 \\
\hline \multirow[t]{23}{*}{15} & 2 & 15 & 2 \\
\hline & & 16 & 3 \\
\hline & & 17 & 2 \\
\hline & & 18 & 1 \\
\hline & & 19 & 7 \\
\hline & & 20 & 7 \\
\hline & & 21 & 3 \\
\hline & & 22 & 4 \\
\hline & & 23 & 3 \\
\hline & & 24 & 1 \\
\hline & & 25 & 3 \\
\hline & & 26 & 3 \\
\hline & & 27 & 4 \\
\hline & & 28 & 2 \\
\hline & & 29 & 3 \\
\hline & & 30 & 3 \\
\hline & & 32 & 1 \\
\hline & & 33 & 3 \\
\hline & & 34 & 5 \\
\hline & & 35 & 3 \\
\hline & & 36 & 7 \\
\hline & & 37 & 2 \\
\hline & & 38 & 1 \\
\hline
\end{tabular}

type). No significant association was found between the OPLL and the control groups.

Several limitations of this study are noted. Firstly, we did not fully cover all genetic variation in the BMP-2 gene analyzed, and only common variants were studied.

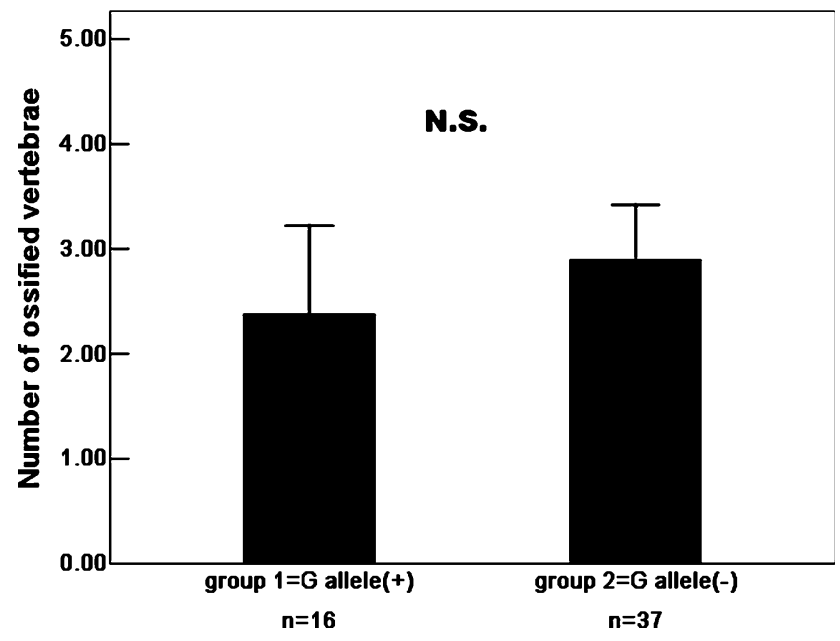

Fig. 3 The number of ossified vertebrae of OPLL patients in each subgroup classified by genotype of the Ser37Ala (T/G). Data are expressed as means (bars) \pm SEM (error bars). The number of patients is shown under each bar. The difference in the number of ossified vertebrae between carriers (group 1) and noncarriers (group 2 ) of deletion $\mathrm{G}$ allele was statistically analyzed. $P>0.05$, N.S., not significant

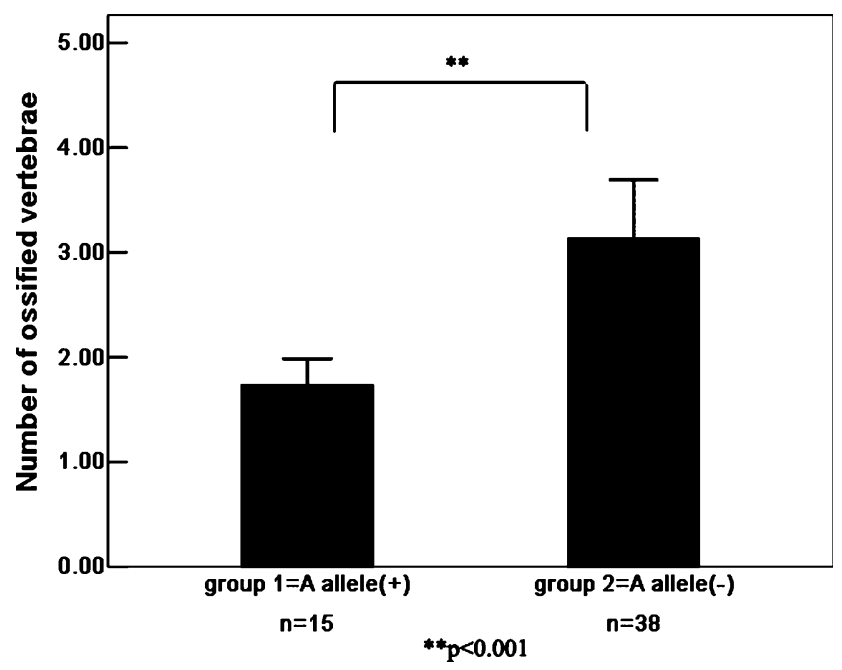

Fig. 4 The number of ossified vertebrae of OPLL patients in each subgroup classified by genotype of the Ser87Ser (A/G). Data are expressed as means (bars) \pm SEM (error bars). The number of patients is shown under each bar. The difference in the number of ossified vertebrae between carriers (group 1) and noncarriers (group 2 ) of deletion A allele was statistically analyzed. $* * P<0.001$

Therefore, if rare variants of this gene were involved in the pathogenesis of OPLL, we might have missed them. Secondly, we only analyzed the patients with OPLL in the cervical spine and excluded the patients with OPLL in the thoracic and/or lumbar spine in the present study. But it is possible that two SNPs in BMP-2 gene affect the bone formation not only in cervical spine, but also in thoracic or lumbar spine in the patients with OPLL. Therefore, based 
on the present study, the authors are going to functionally analyze the difference in the distribution of the BMP-2 polymorphism between the group with OPLL throughout the spine and the group with isolated cervical OPLL in the future. Thirdly, although OPLL of the spine is a common disorder among Japanese and other Asian populations, the present results only drawn in Chinese cannot be generalized to Korean, Japanese, Caucasian populations. Therefore, additional studies are required to elucidate the contribution of two SNPs of BMP-2 gene to the development of ectopic ossification in other ethnic groups.

The functional impact of two SNPs of BMP-2 gene is uncertain, but BMP-2 may lead to increased bone mass in the posterior longitudinal ligament of the cervical spine. The most likely explanation for this is two SNPs variations may yield the alternative transcript and translation with abnormal function and further cause the overexpression of BMP-2 protein in the posterior longitudinal ligament matrix. Several investigations have demonstrated a precise balance to exist between BMP agonists and antagonists, dictating BMP signaling and osteogenesis. The interaction of BMP with the receptor chains is under stringent control of modulator proteins that bind to the ligand and, by blocking it, prevent receptor activation and signaling $[1,3,13]$. These proteins form three distinct families, the noggin, chordin-SOG, and DAN-cerberus families [2]. The overexpression of BMP-2 protein in the posterior longitudinal ligament matrix can augment the signaling activity of endogenously produced BMPs. Further the posterior longitudinal ligament matrix has an imbalance between the expression of BMP-2 agonists and the potential existence of antagonists. Thus BMP-2 protein may be more refractory to inhibitors such as noggin. BMP antagonists are capable of downregulating BMP signal. BMP signal requires the binding of two types of receptor serine/threonine kinases $[4,23]$. Three type I receptors (BMP receptor type IA, BRIA, and type IB, BRIB, and activin type I, ActRI) and two type II (BMP receptor type II, BRII, and activin type II, ActRII) receptors are currently known to bind BMP-2 signal [9, $12,29]$. In addition, previous immunohistochemical study reported that BMPRS and ActRs are highly expressed in ectopic ossified ligaments of patients with OPLL. The receptors were not expressed in a posterior longitudinal ligament from non-OPLL patients. Enhanced expression of BMPRS at the nonossified ligament in OPLL patients suggests that these cells have a greater potential to differentiate into osteogenic cells than ligament cells from non-OPLL patients [38]. Collectively, augmented BMP-2 signal via the high expression of BMPRS and ActRs in the ectopic ossified ligaments suggests that BMP-2 protein is more active and may be tightly involved in the pathological ossification process of OPLL.
In conclusion, the present results demonstrate that the polymorphisms in the BMP-2 gene are not only a factor associated with the occurrence of OPLL, but also a factor related to more extensive OPLL. The "TG" genotype in the Ser37Ala (T/G) polymorphism is associated with the occurrence of OPLL. The "G" allele in the Ser37Ala (T/G) polymorphism is associated with the occurrence of OPLL, but not with the extent of OPLL. The " $G$ " allele in the Ser87Ser $(\mathrm{A} / \mathrm{G})$ polymorphism promotes the extent of OPLL, but does not promote an increased susceptibility to OPLL, whereas the "A" allele in the Ser87Ser (A/G) polymorphism restricts ectopic ossification at least in Chinese subjects. The current studies will benefit to clarify the molecular etiology of OPLL. The understanding of the molecular etiology of OPLL will lead to the development of new therapeutic approaches to the disease.

Acknowledgments The authors thank the DNA donors for making this study possible, and base research — clinic foundation (2007JL38), Capital Medical University (CMU).

Open Access This article is distributed under the terms of the Creative Commons Attribution Noncommercial License which permits any noncommercial use, distribution, and reproduction in any medium, provided the original author(s) and source are credited.

\section{References}

1. Balemans W, Van Hul W (2002) Extracellular regulation BMP signaling in vertebrates: a cocktail of modulators. Dev Biol 250:231-250

2. Garcia Abreu J, Coffinier C, Larrain J et al (2002) Chordin-like $\mathrm{CR}$ domains and the regulation of evolutionarily conserved extracellular signaling systems. Gene 287:39-47

3. Harland RM (2001) Developmental biology. A twist on embryonic signaling. Nature 410:423-424

4. Heldin CH, Eriksson U, Ostman A (2002) New members of the platelet-derived growth factor family of mitogens. Arch Biophys 398:284-290

5. Kawaguchi H, Kurokawa T, Hoshino Y et al (1992) Immunohistochemical demonstration of bone morphogenetic protein-2 and transforming growth factor-[beta] in the ossification of the posterior longitudinal ligament of the cervical spine. Spine 17(Suppl):33-36

6. Koga H, Hayashi K, Taketomi E et al (1996) Restriction fragment length polymorphism of genes of the [alpha]2(XI) collagen, bone morphogenetic protein-2, alkaline phosphatase, and tumor necrosis factor-[alpha] among patients with ossification of posterior longitudinal ligament and controls from the Japanese population. Spine 21(4):469-473

7. Koga H, Sakou T, Taketomi E et al (1998) Genetic mapping of ossification of the posterior longitudinal ligament of the spine. Am J Hum Genet 62:1460-1467

8. Li Z, Zhang G, Sheng C (1999) The incidence of the ossification of the posterior longitudinal ligament in northern region of China. Chin J Spine Spinal Cord 9(5):285-286

9. Liu F, Ventura F, Doody J et al (1995) Human type II receptor for bone morphogenetic proteins (BMPs): extension of the twokinase receptor model to the BMPs. Mol Cell Biol 15:3479-3486 
10. Maeda S, Ishidou Y, Koga $\mathrm{H}$ et al (2001) Functional impact of human collagen $\alpha 2$ (XI) gene polymorphism in pathogenesis of ossification of the posterior longitudinal ligament of the spine. J Bone Miner Res 16:948-957

11. Maeda S, Koga H, Matsunaga S et al (2001) Gender-specific haplotype association of collagen alpha-2(XI) gene in ossification of the posterior longitudinal ligament of the spine. J Hum Genet $46: 1-4$

12. Massague J (1998) TGF- $\beta$ signaling transduction. Annu Rev Biochem 67:753-791

13. Massague J, Chen YG (2000) Controlling TGF- $\beta$ signaling. Genes Dev 14:627-644

14. Matsunaga S, Sakou T (1997) Epidemiology of ossification of the posterior longitudinal ligament. In: Yonenobu K, Sakou T, Ono K (eds) OPLL: ossification of the posterior longitudinal ligament. Springer-Verlag, Tokyo, pp 11-17

15. Matsunaga S, Yamaguchi M, Hayashi K et al (1999) Genetic analysis of ossification of the posterior longitudinal ligament. Spine 24(10):937-939

16. Nakamura I, Ikegawa S, Okawa A et al (1999) Association of the human NPPS gene with ossification of the posterior longitudinal ligament of the spine (OPLL). Hum Genet 104:492-497

17. Ogata N, Koshizuka Y, Miura T et al (2002) Association of bone metabolism regulatory factor gene polymorphisms with susceptibility to ossification of the posterior longitudinal ligament of the spine and its severity. Spine 27(16):1765-1771

18. Okawa A, Nakamura I, Ikegawa S et al (1998) Mutation in NPPS in a mouse model of ossification of the posterior longitudinal ligament of the spine. Nat Genet 19:271-273

19. Onji Y, Akiyama H, Shimomura Y et al (1967) Posterior paravertebral ossification causing cervical myelopathy: A report of eighteen cases. J Bone Joint Surg [Am] 49:1314-1328

20. Ono K, Ota H, Tada K (1977) Ossified posterior longitudinal ligament: a clinicopathological study. Spine 2:126-138

21. Padgett RW, Wozney JM, Gelbart WM (1993) Human BMP sequences can confer normal dorsal-ventral patterning in the Drosophila embryo. Proc Natl Acad Sci USA 90:2905-2909

22. Sakou T, Taketomi E, Matsunaga $S$ et al (1991) Genetic study of ossification of the posterior longitudinal ligament in the cervical spine with human leukocyte antigen haplotype. Spine 16:12491252

23. Shi Y, Massague J (2003) Mechanisms of TGF- $\beta$ signaling from cell membrane to the nucleus. Cell 113:685-700

24. Styrkarsdottir U, Cazier J-B, Kong A et al (2003) Linkage of osteoporosis to chromosome 20p12 and association to BMP2. PLoS Biol 1(3):351-360

25. Tahara M, Aiba A, Yamazaki M et al (2005) The extent of ossification of posterior longitudinal ligament of the spine associated with nucleotide pyrophosphatase gene and leptin receptor gene polymorphisms. Spine 30(8):877-880

26. Taketomi E, Sakou T, Matsunaga S et al (1992) Family study of a twin with ossification of the posterior longitudinal ligament in the cervical spine. Spine 16:1249-1252

27. Takuwa Y, Matsumoto T, Kurokawa T et al (1985) Calcium metabolism in paravertebral ligamentous ossification. Acta Endocrinol 109:428-432

28. Tanaka T, Ikari K, Furushima K et al (2003) Genomewide linkage and linkage disequilibrium analyses identify COL6A1, on chromosome 21 , as the locus for ossification of the posterior longitudinal ligament of the spine. Am J Hum Genet 73:812-822

29. Ten Dijke P, Yamashita H, Sampath TK et al (1994) Identification of type I receptor for osteogenic protein-1and Bone morphogenetic protein-4. J Biol Chem 269:16985-16988

30. Terayama K (1989) Genetic studies on ossification of the posterior longitudinal ligament of the spine. Spine 14:1184-1191

31. Tsuyama N (1984) Ossification of the posterior longitudinal ligament of the spine. Clin Orthop Relat Res 184:71-84

32. Valdes AM, Hart DJ, Jones KA et al (2004) Association study of candidate genes for the prevalence and progression of knee osteoarthritis. Arthritis Rheum 50(8):2497-2507

33. Valdes AM, Van Oene M, Hart DJ, Surdulescu GL et al (2006) Reproducible genetic associations between candidate genes and clinical knee osteoarthritis in men and women. Arthritis Rheum 54(2):533-539

34. Wang EA, Rosen V, Cordes P et al (1988) Purification and characterization of other distinct bone-inducing factors. Proc Natl Acad Sci U S A 85:9484-9488

35. Wozney JM, Rosen V, Celeste AJ et al (1988) Novel regulators of bone formation: molecular clones and activities. Science 242:1528-1534

36. Yamaguchi A, Katagiri $T$, Ikeda $T$ et al (1990) Recombinant human BMP-2 (rhBMP-2) stimulates osteoblastic maturation and inhibits muscle cell differentiation in the pluripotential rat osteoblastic cell line ROB-C26. J Bone Miner Res 5(Suppl 2):211

37. Yasui N, Ono K, Yamaura I et al (1983) Immunohistochemical localization of types I, II and III collagens in the ossified posterior longitudinal ligament of the human cervical spine. Calcif Tissue Int 35:159-163

38. Yonemori K, Imamura T, Ishidou Y et al (1997) Bone morphogenetic protein receptors and activin receptors are highly expressed in ossified ligament tissues of patients with ossification of the posterior longitudinal ligament. Am J Pathol 150(4):13351347 